\title{
Intraoperative Lymph Node Management During Non-small Cell Lung Cancer Surgery
}

\author{
Michael R. Gooseman, $\mathrm{MD}^{1}$, and Alessandro Brunelli, $\mathrm{MD}^{2}$ \\ ${ }^{1}$ Department of Cardiothoracic Surgery, Castle Hill Hospital, Hull University Teaching Hospitals NHS Trust, Cottingham, \\ UK; ${ }^{2}$ Department of Thoracic Surgery, St. James University Hospital, Leeds Teaching Hospitals NHS Trust, Leeds, UK
}

The current issue of the Annals of Surgical Oncology publishes an excellent study by Handa et al. ${ }^{1}$ This work supports the use of systematic lymph node dissection in patients with hypermetabolic non-small cell lung cancer (NSCLC) and adds further clarification on what is a highly contentious and debated matter. Lymph node management during lung cancer surgery continues to be the subject of significant debate and analysis in the thoracic surgical community. Lymphadenectomy and lymph node sampling are fundamental in allowing for accurate staging with potential for survival benefit. The various intraoperative techniques relating to lymph node assessment demonstrate a lack of definitive indications regarding the best option. Given this, over the last couple of decades, there have been numerous studies assessing surgical lymph node management with factors such as surgical morbidity, recurrence rate, and long-term survival.

Standard treatment since the 1990s for early NSCLC has been lung resection with associated mediastinal lymph node dissection. Generally, since then, the treatment methods used in this disease have continued to evolve, and the prognosis continues to improve. However, there are still many issues that trigger debate, and lymph node dissection and sampling remains a major issue in respect of surgery. The surgical gold standard in early disease continues to be lobectomy combined with systematic lymph node dissection. ${ }^{2}$ However, as minimally invasive surgery

(C) Society of Surgical Oncology 2021

First Received: 31 March 2021

Accepted: 2 April 2021;

Published Online: 16 April 2021

A. Brunelli, MD

e-mail: alex.brunelli@nhs.net has become the major approach to lung resection, lymph node approach has received further scrutiny. Of course, this is also set alongside the debate regarding the optimal level of pulmonary resection, which may impact on lymphadenectomy approach. There are data to show that sublobar resections, particularly segmentectomy, are appropriate alternatives to lobectomy. ${ }^{3,4}$ A nonlobectomy resection undoubtedly impacts the surgeon's lymph node approach.

While there is increasing focus on the extent of resection and lymphadenectomy, there are ongoing changes in the medical management of NSCLC that will impact on surgical approach and decision-making. The use of immune checkpoint inhibitors and tyrosine kinase inhibitors (TKI) therapy [for patients with epidermal growth factor receptor (EGFR) mutation] in resectable NSCLC is the subject of ongoing trials in both the neoadjuvant and adjuvant settings. Especially in hypermetabolic cancers with a higher propensity to nodal spreading, multimodal approach has become standard of care. Although in principle the approach to nodal dissection should not be affected by preoperative anticancer treatment, a more hostile operating field following immunotherapy or chemoradiation therapy may still influence the surgical dissection. Surgical experience in this setting describes obliterated tissue planes with fibrotic hila and much more difficulty in dissection. ${ }^{5}$ It also appears very difficult to predict which patients will have reactive and inflammatory changes with nodal flare. These factors may have an impact on the way in which lymph nodes are dissected and sampled intraoperatively.

On the other hand, systematic nodal dissection ensures appropriate staging, which is critical for selecting patients for adjuvant treatment. Particularly in the light of emerging 
novel systemic treatments, inaccurate nodal sampling may inappropriately deprive patients of these effective treatments, impacting on their survival. ${ }^{6,7}$

Systematic lymph node dissection is recommended to guarantee proper assessment of mediastinal lymph node status. Despite this, there are those who advocate for lobespecific mediastinal lymph node dissection, which provided the foundation for the work of Handa et al. ${ }^{1}$ The idea is that any lymph node metastasis is likely related to the location of the primary tumour. For those tumours in the upper lobe, there is higher incidence of metastasis within superior mediastinal lymph nodes. Lower-lobe tumours tend to spread to the inferior and subcarinal nodes. There are therefore those who advocate a targeted approach to lymph node dissection such as omission of station 7 dissection in the surgical resection of a NSCLC. ${ }^{8}$ The current study helps emphasize the importance of following a systematic approach to lymph node management. We must make the point that, as per European Society Thoracic Surgeons (ESTS) guidelines, we would always recommend sampling of the subcarinal nodes, even for those specific situations in which a lobe-specific lymphadenectomy is performed (peripheral T1 squamous cell carcinoma). ${ }^{9}$ These recommendations are based on detailed analyses on lobe-specific lymphatic drainage. ${ }^{10,11}$

In any case, subcarinal lymph nodes should always be sampled. One of the most important findings of the Handa et al. ${ }^{1}$ study is the fact that nearly $6 \%$ of patients in the lobe-specific dissection group might have had their metastatic lymph nodes missed by not sampling stations not included in the "lobe-specific stations." In practical terms, this translates into $6 \%$ of patients potentially denied adjuvant systemic treatment.

While more conservative nodal sampling may be acceptable in less invasive forms of cancer (such as those small sized and located peripherally, with non-solid or predominantly nonsolid features, or those with scarce metabolic activity on PET scan), systematic nodal dissection should remain the mainstay of a proper lung cancer operation in the majority of patients to ensure optimal multimodal management of their disease.

DISCLOSURES The authors declare that they have no conflict of interest.

\section{REFERENCES}

1. Handa Y, Tsutani Y, Mimae T, Miyata Y, Ito H, Shimada Y, et al. Systematic versus lobe-specific mediastinal lymphadenectomy for hypermetabolic lung cancer. Ann Surg Oncol. 2021. https://d oi.org/10.1245/s10434-021-10020-2.

2. Howington JA, Blum MG, Chang AC, et al. Treatment of stage I and II non-small cell lung cancer: diagnosis and management of lung cancer, 3rd ed: American College of Chest Physicians evidence-based clinical practice guidelines. Chest. 2013;143(5 Suppl):e278S-313S.

3. Landreneau RJ, Normolle DP, Christie NA, et al. Recurrence and survival outcomes after anatomic segmentectomy versus lobectomy for clinical stage I non-small-cell lung cancer: a propensitymatched analysis. J Clin Oncol. 2014;32(23):2449-55.

4. Altorki NK, Yip R, Hanaoka T, et al. Sublobar resection is equivalent to lobectomy for clinical stage $1 \mathrm{~A}$ lung cancer in solid nodules. J Thorac Cardiovasc Surg. 2014;147(2):754-62. (discussion 762-754)

5. Bott MJ, Cools-Lartigue J, Tan KS, Dycoco J, Bains MS, Downey RJ, Huang J, Isbell JM, Molena D, Park BJ, Rusch VW, Sihag S, Jones DR, Adusumilli PS. Safety and feasibility of lung resection after immunotherapy for metastatic or unresectable tumors. Ann Thorac Surg. 2018;106(1):178-83.

6. Jones DR, Wu YL, Tsuboi M, Herbst RS. Targeted therapies for resectable lung adenocarcinoma: ADAURA opens for thoracic oncologic surgeons. J Thorac Cardiovasc Surg. 2021. https://doi. org/10.1016/j.jtcvs.2021.02.008.

7. Wu YL, Tsuboi M, He J, John T, Grohe C, Majem M, Goldman JW, Laktionov K, Kim SW, Kato T, Vu HV, Lu S, Lee KY, Akewanlop C, Yu CJ, de Marinis F, Bonanno L, Domine M, Shepherd FA, Zeng L, Hodge R, Atasoy A, Rukazenkov Y, Herbst RS; ADAURA Investigators. Osimertinib in resected EGFR-mutated non-small-cell lung cancer. N Engl J Med. 2020;383(18):1711-23

8. Aokage K, Yoshida J, Ishii G, et al. Subcarinal lymph node in upper lobe non-small cell lung cancer patients: is selective lymph node dissection valid? Lung Cancer. 2010;70(2):163-7.

9. Lardinois D, De Leyn P, Van Schil P, Rami Porta R, et al. ESTS guidelines for intraoperative lymph node staging in non-small cell lung cancer. EJCTS. 2006;30(5):787-92.

10. Naruke T, Tsuchiya R, Kondo H, Nakayama H, Asamura H. Lymph node sampling in lung cancer: how should it be done? Eur J Cardiothorac Surg. 1999;16(Suppl 1):S17-24.

11. Ichinose Y, Kato H, Koike T, Tsuchiya R, Fujisawa T, Shimizu N, Watanabe Y, Mitsudomi T, Yoshimura M, Tsuboi M; Japanese Clinical Oncology Group. Completely resected stage IIIA non-small cell lung cancer: the significance of primary tumor location and N2 station. $J$ Thorac Cardiovasc Surg. 2001;122(4):803-8

Publisher's Note Springer Nature remains neutral with regard to jurisdictional claims in published maps and institutional affiliations. 